\title{
AVALIAÇÃO DO TEOR DE FERRO NA FORMAÇÃO DA MACROESTRUTURA E O CONSEQÜENTE EFEITO SOBRE A CONDUTIVIDADE ELÉTRICA DO ALUMÍNIO ELETRO-CONDUTOR
}

\author{
Moreira, A.E.A. ${ }^{1}$ \\ Mercês, M.P. ${ }^{2}$ \\ Machado, P.C. ${ }^{3}$ \\ Santos, W.L.R. ${ }^{4}$ \\ Quaresma, J.M.V.
}

Resumo: O objetivo deste estudo é avaliar o efeito de adições de ferro no alumínio eletro condutor [Al-EC], sem refinador de grão, no que diz respeito à formação da macroestrutura, bem como o tamanho dos grãos, e sua influência sobre a Condutividade Elétrica. Para este estudo, inicialmente elaborou-se a liga cuja composição representativa foi $\mathrm{Al}-0,05 \% \mathrm{Cu}-0,11 \% \mathrm{Fe}$ e que convencionou-se identificar como Al-EC. Esta liga posteriormente foi modificada com os teores: $[0,40 ; 0,80 ; 1,10$ e 1,60$] \%$. As ligas foram fundidas em um cadinho de carbeto de silício, em um forno do tipo mufla e vazadas em um molde no formato U. Termopares do tipo $\mathrm{K}$ foram utilizados para verificar a temperatura de vazamento. Os resultados mostram que o ferro ao controlar o crescimento do grão apresenta um efeito de transição para teores no entorno de $0,8 \%$, apresentando o maior tamanho de grão sem, no entanto, afetar a capacidade mecânica e de conduzir eletricidade.

Palavras-chave: macroestrutura, tamanho de grão, propriedades mecânicas e elétricas.

Abstract: The objective of this study is to evaluate the effect of additions of iron in electro conducting aluminum [Al-EC], without grain refiner, that respect to the formation of the macrostructure, as the grain size, and its influence on the Electric Conducting. Initially, for this study it was elaborated a alloy that represent as $\mathrm{Al}-0,05 \% \mathrm{Cu}-0,11 \% \mathrm{Fe}$ and stipulated to identify as Al-EC. Later, this alloys was modified with contents: $[0,40 ; 0,80 ; 1,10$ and $1,60] \%$. The alloy had been casting in crucible silicon's Carbon , in an oven of the type muffle and casting in a mold in " $U$ " format .Thermocouples of $\mathrm{K}$ type had been used to verify the temperature of casting. The results show that the iron when controll the growth of the grain presents a effect of transistion for contents between of $0,8 \%$, presenting the biggest size of grain without, however, affect the mechanics capacity and electricity coducting.

Keywords: macrostructure; size of grain; mechanical and electric properties.

Universidade Federal do Pará, ${ }^{1}$ aline@gmail.com; ${ }^{2}$ manu@hhotmail.com; ${ }^{3}$ pcmufpa@yahoo.com.br, ${ }^{4}$ wlrs@ufpa.br, ${ }^{5}$ quaresma@fem.unicamp.br 


\section{INTRODUÇÃO}

O refinamento de grão é uma técnica importante para a melhoria dos produtos fabricados a partir do alumínio. Estruturas equiaxiais tornam as propriedades mecânicas do material uniforme, melhorando a usinabilidade e os aspectos superficiais. Os refinadores normalmente utilizados são Al-Ti ou Al$\mathrm{Ti}-\mathrm{B}$, entretanto, estudos desenvolvidos no GPEMAT/UFPA constataram que o $\mathrm{Si}$ apresenta características que podem promover o refinamento do grão de ligas 6101, Al-0,06\%Mg-[0,40; 0,80 e $1,20] \% \mathrm{Si}, \quad$ com solidificação unidirecional horizontal [MAIA et al, 2005], por outro lado Backerud e Johnsson (1996) e Zhiyong et al (2003) desenvolveram estudo com teores variados de $\mathrm{Si}$ e constataram sua capacidade de refinar grão. Estudos de Zhang et al (2005) mostram o efeito do teor de ferro, em alumínio eletrocondutor como refinador de grão, como visto nas macro estruturas da Figura 1.

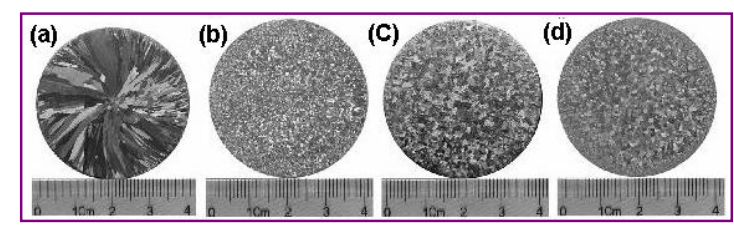

Figura 1 - Evolução da macroestrutura do Al-EC com variação do teor de Fe: (a) Al-EC; (b) 0,5\%Fe; (c) $0,8 \% \mathrm{Fe}$; (f) 1,2 $\% \mathrm{Fe}$. [ZHANG et al, 2005].

Estes benefícios são cruciais na industria de condutores elétricos, visto que se almeja ligas capazes de serem deformadas sem apresentar defeitos estruturais e sem comprometer as características mecânicas e elétricas do perfil deformado.

Baseado em estudos realizados sobre a influencia de teores de $\mathrm{Fe}$ na formação da macroestrutura de alumínio, este artigo pretende contribuir com estas considerações ao apresentar um estudo em que a liga $\mathrm{Al}-0,05 \% \mathrm{Cu}-0,10 \% \mathrm{Fe}$, modificada com os teores de $\mathrm{Fe}(0,40$; $0,80 ; \quad 1,10$ e 1,60$) \%$ apresenta modificação na formação da macroestrutura e, além disso, avalia as conseqüências nas características mecânicas e elétricas do produto final.

\section{DESENVOLVIMENTO}

\subsection{MATERIAIS E MÉTODOS}

Para realização do estudo, foram confeccionadas amostras com as ligas Al$0,05 \% \mathrm{Cu}-0,10 \% \mathrm{Fe}$, e com as ligas Al$0,05 \% \mathrm{Cu}-[0,40 ; 0,80 ; 1,10$ e 1,60$] \% \mathrm{Fe}$. As ligas foram elaboradas em um forno tipo mufla, marca BRASIMET, utilizando-se um cadinho de grafite, revestido interiormente por uma pintura protetora de caulim e vazadas em coquilha de aço-carbono na forma de "U", como pode ser vista na Figura 2.

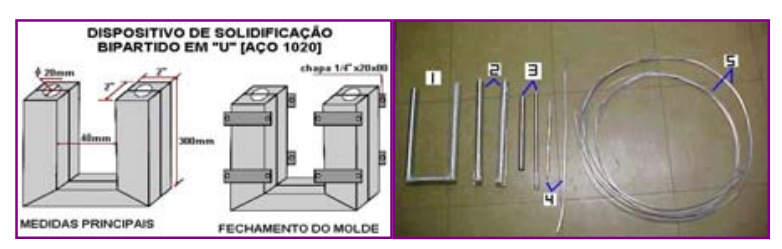

Figura 2 - O molde em forma de "U" e os perfis obtidos.

Este molde permitiu a obtenção de dois corpos-de-prova, por liga, no formato cilíndrico de $20 \mathrm{~mm}$ de diâmetro e $230 \mathrm{~mm}$ de comprimento, aproximadamente. A análise metalográfica foi desenvolvida em um microscópio LEICA acoplado a um micro computador de última geração equipado com software para análise das imagens. As amostras, para esta finalidade, foram retiradas da parte inferior do perfil "U" obtido, e os braços laterais do perfil foram usinados para o diâmetro de 10 $\mathrm{mm}$. Após a usinagem, as amostras foram laminadas até o diâmetro de $3,98 \mathrm{~mm}$, em um laminador com canais circulares e posteriormente trefiladas até o diâmetro de $2,90 \mathrm{~mm}$ em um trefilador industrial marca TECALSA modelo 450EE. Desta forma foi possível realizar ensaios de resistividade, em um micro ohmímetro marca MEGABRAS modelo MPK 2000e, para a obtenção da condutividade elétrica. Os ensaios de tração foram realizados em uma máquina KRATOS, 
modelo IKCL1-USB com velocidade de $10 \mathrm{~mm} / \mathrm{min}$, temperatura de $25^{\circ} \mathrm{C}$, uma pré-carga $220 \mathrm{~N}$ e umidade relativa $70 \%$. $\mathrm{O}$ ensaio foi realizado segundo a norma NBR-6810-1991(corpo-de-prova

laminado), para a análise de limite de resistência à tração (LRT) e alongamento em corpo-de-prova de $150 \mathrm{~mm}$.

Cada liga recebeu injeção de argônio durante 40 segundos, antes do vazamento e ao atingir a temperatura de $750^{\circ} \mathrm{C}$, como forma de se minimizar a ocorrência de poros pela presença de hidrogênio, foi vazada. A análise química foi realizada em um Espectrômetro Óptico, os resultados encontram-se relacionados na Tabela 1 , abaixo.

Tabela 1 - Composição química das ligas estudadas

\begin{tabular}{|c|c|c|c|c|c|}
\hline \multicolumn{3}{|c|}{ Liga } & Si $(\%)$ & $\operatorname{Mg}(\%)$ & $\mathrm{Fe}(\%)$ \\
\hline \multicolumn{3}{|c|}{$\mathrm{Al}-0,05 \% \mathrm{Cu}-0,10 \% \mathrm{Fe}$} & 0,0500 & 0,00089 & 0,1122 \\
\hline \multicolumn{3}{|c|}{ Al-0,05\%Cu-0 $4 \% \mathrm{Fe}$} & 0,0534 & 0,0020 & 0,3577 \\
\hline \multicolumn{3}{|c|}{$\mathrm{Al}-0,05 \% \mathrm{Cu}-08 \% \mathrm{Fe}$} & 0,0531 & 0,0000 & 0,7950 \\
\hline \multicolumn{3}{|c|}{$\mathrm{Al}-0,05 \% \mathrm{Cu}-1,10 \% \mathrm{Fe}$} & 0,0502 & 0,0000 & 1,1392 \\
\hline \multicolumn{3}{|c|}{$\mathrm{Al}-0,05 \% \mathrm{Cu}-1,6 \% \mathrm{Fe}$} & 0,0571 & 0,0000 & 15677 \\
\hline $\mathrm{Cu}(\%)$ & Ti(\%) & & $\%$ & Al\%) & Outros (\%) \\
\hline 0,0463 & 0,0011 & & 015 & 99,76 & 0,0172 \\
\hline 0,0502 & 0,0009 & & 011 & 99,49 & 0,0428 \\
\hline 0,0508 & 0,0006 & & 015 & 99,00 & 0,0936 \\
\hline 0,0497 & 0,0007 & & 015 & 98,65 & 0,1054 \\
\hline 0,0481 & 0,0007 & & 015 & 98,21 & 0,1150 \\
\hline
\end{tabular}

\subsection{RESULTADOS E DISCUSSÕES}

A análise metalográfica permitiu avaliar o efeito do ferro na liga Al$0,05 \% \mathrm{Cu}-0,10 \% \mathrm{Fe}$. A Figura 3 mostra a macrografia das ligas.

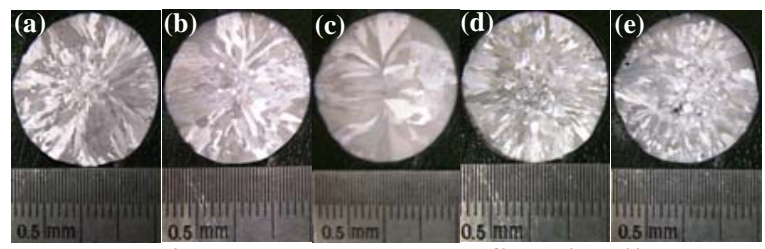

Figura 3 - Macrografias das ligas estudadas, na seqüência: (a) $0,10 \%$, (b) $0,4 \%$, (c) $0,8 \%$, (d) $1,1 \%$, (e) $1,6 \%$ de Fe.

A Figura 3(a) mostra a macrografia da liga $\mathrm{Al}-0,05 \% \mathrm{Cu}-$ $0,10 \% \mathrm{Fe}$, e é possível notar grãos colunares na periferia e, no centro, grãos equiaxiais. Á medida que se adiciona ferro, em (b), os grãos colunares diminuem de tamanho e a estrutura equiaxial se faz menos presente, em (c) os grãos estão completamente colunares, em (d) a adição de ferro diminuiu o tamanho dos grãos, da periferia, que nos teores anteriores tendiam a manter os grãos colunares, agora com $1.6 \%$ de ferro mostra grãos mais finos, ou seja, onde se localizavam grãos mais alongados e espessos agora dão forma a grãos de tamanho menor, embora ainda colunares, mas de largura menor, a mesma característica é observada em (e) onde o centro dá lugar a grãos mais refinados e em maior quantidade. Estudos de Zhang et al (2005) mostraram a tendência do ferro em refinar os grãos em matriz de alumínio, porém o teor de cobre era inexistente. Este fato permite inferir que a combinação $\mathrm{Cu} / \mathrm{Fe}$ tende a interferir na estrutura bruta de fusão destas ligas.

$\mathrm{O}$ gráfico da Figura 4 mostra o comportamento do tamanho de grão em relação o teor de ferro e, no qual o valor maior representa a medida do TG para a liga com $0,8 \%$ de ferro e cuja macro estrutura apresenta-se completamente colunar. Para a medida do TG foi adotada a técnica dos interceptos NBR 1323/1990.

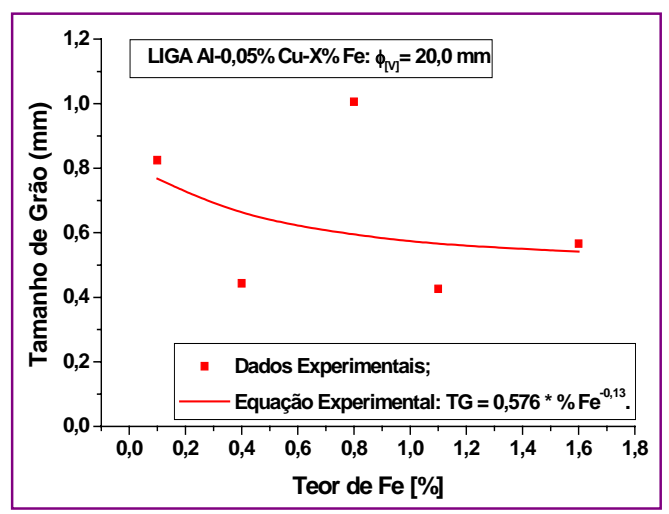

Figura 4- Variação do tamanho de grão em função do teor de ferro.

Uma outra maneira de medir o TG pode ser feita pela diferença das áreas colunar e coquilhada produzidas em função do teor de ferro nas estruturas das ligas. Neste caso a metodologia adotada no processo de medição das áreas foi o existente no Software Motic Images Plus 2.0. Os resultados encontram-se listados na Tabela 2 e plotados na Figura 5. 
Tabela 2 - Relação de áreas das macro estruturas

\begin{tabular}{|c|c|c|c|c|c|c|}
\cline { 2 - 7 } \multicolumn{1}{c|}{$\%$ Fe } & \multicolumn{3}{c|}{ Área $\left(\mathrm{mm}^{2}\right)$} & \multicolumn{3}{c|}{ Porcentagem $(\%)$} \\
\cline { 2 - 7 } \multicolumn{1}{c|}{} & Coq. & Colun. & Total & Coq. & Colun. & Total \\
\hline 0,1 & 31.2 & 273.4 & 304.6 & 10.3 & 89.7 & 100 \\
\hline 0,4 & 32.9 & 271.7 & 304.6 & 10.8 & 89.2 & 100 \\
\hline 0,8 & 0 & 304.6 & 304.6 & 0 & 100 & 100 \\
\hline 1,1 & 71.3 & 233.3 & 304.6 & 23.4 & 76.6 & 100 \\
\hline 1,6 & 109.2 & 195.4 & 304.6 & 35.9 & 64.1 & 100 \\
\hline
\end{tabular}
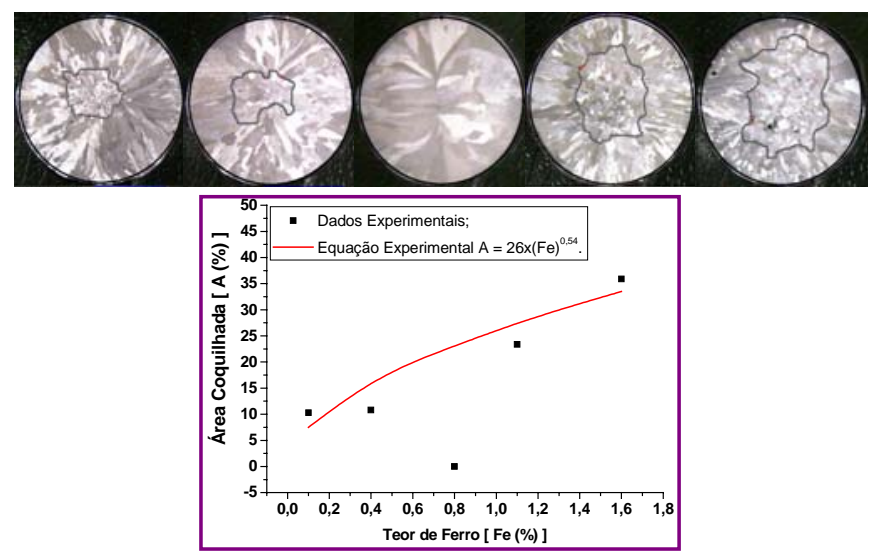

Figura 5 - Arranjo demonstrativo da evolução da estrutura coquilhada em função do teor de Ferro para as ligas em estudo.

Os gráficos da Figura 6 mostram os resultados de ensaio de tração para cada teor estudado em função da quantidade relativa dos grãos coquilhados. A partir dos ensaios mecânicos foi possível obter o limite de resistência à tração e alongamento.
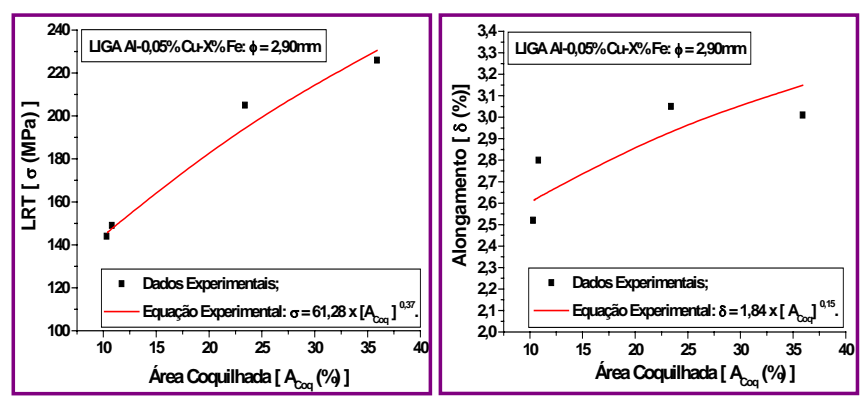

Figura 6 - Resultados dos Ensaios de

Tração: [A] LRT e em [B] Alongamento para o fio $2,90 \mathrm{~mm}$ em função da quantidade relativa dos grãos coquilhados.

A partir das curvas da Figura 6 é possível verificar que o limite de resistência à tração aumenta sem perder a capacidade de deformar à medida que se adiciona ferro. Isto pode estar ocorrendo por dois motivos: i) $\mathrm{O}$ binário $\mathrm{Cu} / \mathrm{Fe}$ aparentemente contribui para produzir zonas centrais coquilhadas que realmente estarão sujeitas a todas às deformações a partir do diâmetro de $10 \mathrm{~mm}$; ii) Maiores regiões coquilhadas contribuem para melhorar a "trabalhabilidade" da liga, elevando seu LRT por maior capacidade de encruar sem, no entanto fragilizar-se.

Os dados elétricos medidos foram resistências elétricas para cada teor de liga. Após a obtenção desses dados, foi necessária a correção da temperatura do ensaio, uma vez que influencia na resposta elétrica do material, a norma NBR 6814/1985 traz cálculos de correção para uma temperatura ambiente a $20^{\circ} \mathrm{C}$.

$$
R_{t_{0}}=\frac{R_{t}}{1+\alpha_{t_{0}}\left(t-t_{0}\right)}
$$

Em seguida, o cálculo de resistividade elétrica é realizado, segundo a norma NBR 6815/1981.

$$
R m=R C * m c
$$

Através dos resultados da condutividade elétrica da Figura 7 , relativos às ligas $\mathrm{Al}-0,05 \% \mathrm{Cu}-(0,10 ; 0,4$; $0,8 ; 1,1$ e 1,6$) \% \mathrm{Fe}$, observa-se que à medida que o binário $\mathrm{Cu} / \mathrm{Fe}$ é mais presente na liga, pelo crescente teor de ferro, a capacidade de conduzir eletricidade diminui. Particularmente para os teores maiores que $0,8 \%$, caindo a baixo de 57 IACS. Estes resultados permitem inferir que teores de ferro maiores que $0,8 \%$, que contribuem na formação da zona central coquilhada interferem no LRT e na "trabalhabilidade" positivamente, não agem da mesma forma quando se trata da condutividade elétrica. Neste caso melhor será adotar-se teores menores que $0,8 \%$ de ferro com os quais podem ser obtidos valores para a condutividade elétrica, compatíveis aos das ligas da série 6000 quando submetidas à têmpera, da ordem de 58 IACS (Hand Book, vol. 2). 


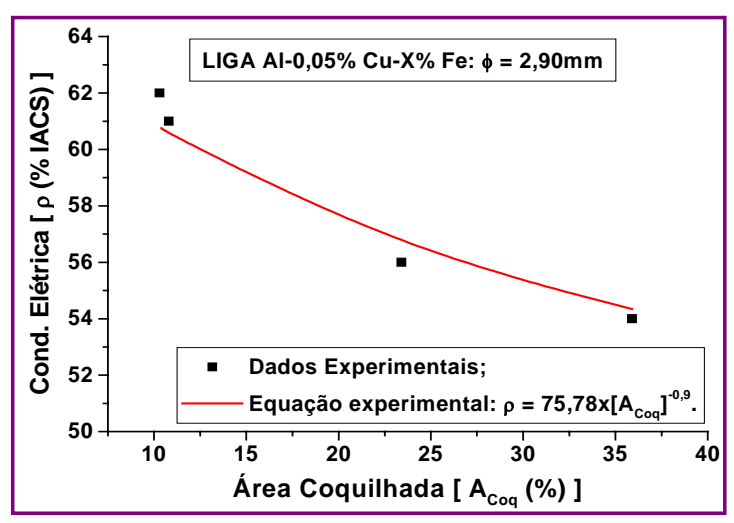

Figura 7 - Condutividade elétrica da liga $\mathrm{Al}-0,05 \% \mathrm{Cu}-(0.4 ; 0,6 ; 0,8 ; 1,2$ e 1,6$)$ $\% \mathrm{Fe}$ para o fio de diâmetro $2,90 \mathrm{~mm}$ em função da quantidade relativa dos grãos coquilhados.

\section{CONCLUSÃO}

O teor de $0,8 \%$ de ferro parece atuar como uma referência. Teores a cima ou a baixo associados com $0,05 \%$ de Cobre tendem a proporcionar, nas condições experimentais impostas, o surgimento de uma zona central coquilhada.

A partir da análise da macroestrutura das ligas estudadas, notase que a presença do binário $\mathrm{Cu} / \mathrm{Fe}$ cada vez mais marcante pelo maior teor de ferro, atua de forma positiva quanto ao LRT e à "trabalhabilidade", por contribuir com a formação da zona central coquilhada. Entretanto, este desempenho não se traduz quando se trata da Condutividade Elétrica.

A avaliação do ponto de vista da propriedade elétrica melhor será adotar-se teores de ferro menores que 0,8\%, quando se tem boa performance mecânica associada à excelente performance elétrica, podendo mesmo a ser compatíveis às ligas de alumínio da série 6000 , tratadas termicamente.

\section{AGRADECIMENTOS}

O grupo GPEMAT entende ser importante demonstrar o sincero agradecimento à Universidade Federal do Pará pela disponibilidade de espaço físico, ao GRUPO ALUBAR e a ELETRONORTE pelo apoio financeiro, parceria e incentivo ao desenvolvimento de estudos científicos e a FADESP e a FUNPEA que gerenciam essas parcerias entre os grupos.

\section{BIBLIOGRAFIA}

ABNT, NBR 6810 - Fios e cabos elétricos tração à ruptura em componentes metálicos. - Especificação. São Paulo, 1981.

NBR 5118 - Fios de alumínio nus de seção circular para fins elétricos. Especificação. São Paulo, 1985.

NBR 6814/1985 - Ensaio de resistência elétrica. Especificação. São Paulo, 1985.

. NBR 6815/1981 - Fios e Cabos elétricos - Ensaio de determinação da resistividade em componentes metálicos - método do ensaio.

\section{ASM SPECIALITY HANDBOOK.}

Aluminum and Aluminum Alloys. Ed. United States of America: ASM International, 1998.p.68-69,688-689.

ASM HANDBOOK. Volume 2 Properties and Selection Nonferrous Alloys and Special - Purpose Materials. 1998. p. 421- 42.

Backerud, L., Johnsson, M. The relative importance of nucleation and growth mechanisms to control grain size in various aluminum alloys. Light Met 1996;27(4):679-85.

Maia, R. F. da S., Moreira, A. E. A., de Sá, F. A., da Silva, M. B., Quaresma, J. M. do V. - Relação entre a Deformação e 
as Estruturas de Ligas Al-Mg-Si

Solidificadas Unidirecionalmente, 2005.

Zhiyong, L., Mingxing, W., Yonggang, W. et al.. Effect of silicon on grain refinement of aluminum produced by electrolysis. Mater Trans 2003; 44(10): 2157-62.

\section{Nomenclaturas}

$\mathrm{R}_{\mathrm{t} 0} \quad$ Resistência do condutor corrigida à temperatura de $20^{\circ} \mathrm{C}$, em $\Omega$;
$\mathrm{R}_{\mathrm{t}} \quad$ Resistência do condutor medida a uma determinada temperatura, em $\Omega$;

$\alpha_{t 0} \quad$ Coeficiente de temperatura da resistência do condutor à temperatura de $20^{\circ} \mathrm{C}$, em ${ }^{\circ} \mathrm{C}^{-1}$;

$\mathrm{t}$ Temperatura qual foi efetuada a medição, em ${ }^{\circ} \mathrm{C}$;

$\mathrm{t}_{0} \quad$ Temperatura especificada $-20^{\circ} \mathrm{C}$.

$R c$ Resistência elétrica do corpo de prova por unidade de comprimento a $20^{\circ} \mathrm{C}$, em $\Omega / \mathrm{m}$;

$m c$ Massa do corpo de prova por unidade de comprimento, em $\mathrm{g} / \mathrm{m}$. 